calcul de la dépense, on s'est basé sur' le nombre des places de voyageurs. Ce nombre a pu ôtre déterminé avé une approximation suffisante d'aparès le nombre ct le pouds des véhicules, en admellant dune parl avec la stalisticuo des chemins de fer que la lare des wagons s'elève à un quart de tonne par place de voyageur" et, d'autre part, en se basant sur le fait d'expéricnce que le nombre des places est exprimé par" la moibé du poids du tram ; chaque lonne do tram peut done otre comptée pour deux places de voyageurs. C'esl nalurelicment les poids des trains d'hrver qui ont servi de base au cajcul.

Pour le chauffage on peut admolltro uno puissance dépensée de 0,156 knlowalt par' place, d'apres les cxpén"lences failes sur lo chemin do for électrirue de Fribourn à Morat, sur lequel 7,5 kilowalts suffisont pour des. wagons de 18 places. En admettanu cuc le temps de marche dost apparens cto chauffage dure 7 heun'es par jour, co qui esh la monlié du temps toral de travall d'un wagon, on déduit que, pour chaque place, il est nécessair"e do fournin", par jour, une énergie de

$$
\frac{0,156 \times 7}{0,736}=1,48 \text { cheval-heure, }
$$

soit en chiffres ronds 1,5 chcval-heuro.

Pour l'éclairage, on s'est basé sur une intonsité de 2 bougias pary place, et ce qui représente prour les lampes ordinaures une pussance de 7 watts ; comme, cn oulre, los Chemins de fer fédéraux accusent un rendement moyen de 0,3 pour' cet éclarrage, il s'ensurt que chaqua pace exige unepuissance de

$$
\frac{0,007}{0,3 \times 0,736}=0,031 \text { cheval-heure, }
$$

ce qui représente poun un éclairage de 8 houres par jour, un travail de

$$
0,031 \times 8=0,25 \text { cheval-heure environ }
$$

Les résultats de tous les calculs d'énergie sont consignés dans une sunte de tableaux, qui indiquent pour chacun des tionçons d'une môme ligne, différonts comme nature d'exploitation, la longucur du parcours, le poids moyen des trains et le nombre des trains de chaque catégoric, les tonnes transporlées, le lravail à dépenses par tonne el par course, le: travail eventuellement disponble en pento, ic nombre d'es démarrages et le travail total de roulement qui en résulte par tonne-kilomelre. Pour donner une idéc de li minutie da ce travail, disons que la ligne Goneve-Berne a été partagée en tronçons comme suit : Geneve-Mornges, Morges-Lausanne, Lausanne-Palézieux, Palézieux-Romonl, Romont-Fribourg, Fribourg-Berne.

Il en résulte pour l'énergie nécessaire en un jour d'été, pour l'ensemble des chemins de ler suisses à vapeur, l'énergie étant mesurée à la jante des roues motrices, les nombres suivants : pour la traction conformément à l'horaire environ 1150000 chevaux-heure (les Chemins de fer fétlér raux et les lignes exploitées par eux forment les $2 / 3$ do co nombre, les Chomins de fer fédéraux et lo Gohhard onsemble les 90 pour 100 de ce nombre) ; il y a lieu d'ajouler' pour" les manœuvres dans les gares environ 50000 chevaux-heures, ce qui porte l'énergié nécessaire à 1200000 chevaux-heures.

En été, le chaufîag' est nul, l'éclairage insilgnifiant.

Enomęons ici quelques considérations suggérées par ces résultats :

En admettant un système d'exploilation qui ne pcrmelle aucune récupération d'émer"gie dans les descentes, nous avons donc à fournir chaque jourr 1200000 chevamx-heures aux jantes des roues motrices. Quant au travail à fournir journellement par les stations centrales, il dépend du systèmo adopté et de son rendement, Ce système ne peut pas encor'a etre déterminé pour le moment, mais l'on peut prón voir copondant qùl doil, ôtro possible, même avec le système

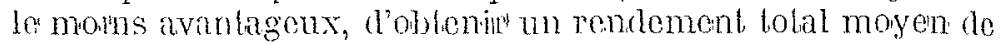
45 pour 100 entre lo braval ublisable aux jantus de l'encrgie foumie nar les turibnnes des stalions primarres. Calculons avec 10 pour 100 senlement. It nous semble que ce rondement lient largement comple des perles qui se produisent

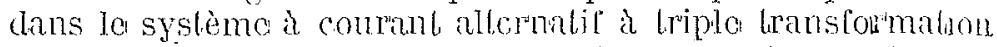
célévation de la tension dans la station contrale, abajssement, de la lonsion aux als cu frolloy el nouvel abarisso-

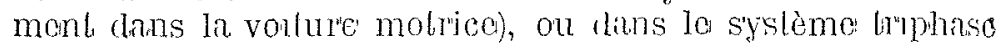
aves transformatom on combnu el baltories d'accumulateurs. Les twobines devront donc fournir joulnellement 3000000 do chevaux-hcuires.

Fun nógligeanl, pour le moment, les reserves hydraulicues a constituer pondaub los dufrécentes saisons do l'anmée of

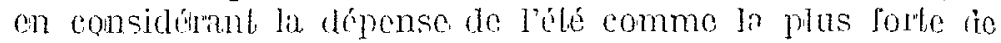
colles rui peuvent so produile, ese 3000000 de chevauxhoures cxigariticnle une pussane continue de 125000 chevanu pendant 24 hewes par jour.

Ils semble rue les forpes hytmuliques actarloment disponibles en Sulsso dípassent ces 125000 chexaux. İun des plus récents projets éludus serant capable à lui seul do fournir le $1 / 5$ de cetice pussanon. Mas il no faul pas oublien" fue

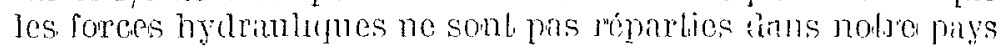

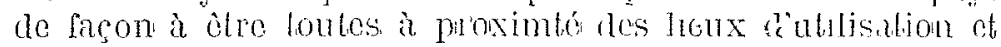
qu'en ouluc, bales les forces hytratulaues ne conviennent.

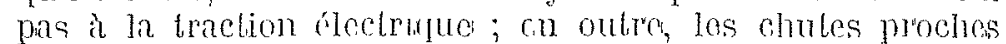
dé la fronlèere ne semblent pas proptes à citre uliliscés pour le service des chemins de: for". II n'esl gas superflu de chercher les moyons de rédure la pursance nécessadio. Fin pro-

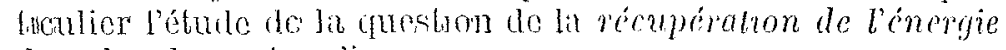
dans les descentes simpose.

(1 suivre.)

\section{La Distribution de l'Énergie Électrique dans la Ville de Grenoble}

(Suite)

Conférence faite le 28 mai 1906 , devant la Société pour le développement de l'Enseignement technique près l'Unversité de Grenoble, par M. F. Jenny, Ingenieur, chef du service electrique de la ville de Grenoble.

\section{SECTEUR MUNICIPAL}

La distribution générale de l'Energie Electrique sur tout le territoire de la commune de Grenoble est donc réalisée par des courants alternatifs triphasés à 3 fils et 5o périodes.

Des feeders souterrains à 5000 volts distribuent le courant dans les différents postes où il est transformé à I 25 volts.

De là, le courant à basse tension est divisé en 2 parties distinctes ; l'éclairage particulier, et l'éclairage public. Le double réseau est souterrain dans le centre de la ville et dans les avenues où il était impossible de réaliser différemment le transport ; il est aérien dans la périphérie et dans la banlieue, partocit où ce mode d'installation a pu être adopté.

Toutefois, dans la banlieue, pour des raisons que nous indiquerons tout à l'heure, le réseau d'éclairage public n'est pas complètement distinct đu réseau particulier; il est constitué par un simple fil d'allumage et d'extinction.

Réscau prinaire. Feeders. - Les 7 panneaux de feeders dont il a été question précédemment, commandent chacun un câble à 5000 volts alimentant séparément un quartier de la ville. Les 3 premiers distribuent l'énergie électrique au centre et à l'est, c'est-ä̀dire dans la partie de 
la ville où la population est la plus dense. Ces 3 feeders, quoique établis pour fonctionner séparément, peuvent être reliés entre eux, environ au centre de leur rayon d'action, et ce, par une simple mancuvre d'interrupteurs et de broches, montés dans trois postes de transformateurs. Cette disposition présente le très grand avantage de permettre l'alimentation de 2 feeders par un seul, au cas où accident se produirait sur l'un d'eux. Le schéma de la figure 8 cijointe indique clairement comment ces couplages peuvent être exécutés. (Pour simplifier le dessin, les 3 fils d'un même circuit sont représentés par un seul et même trait).

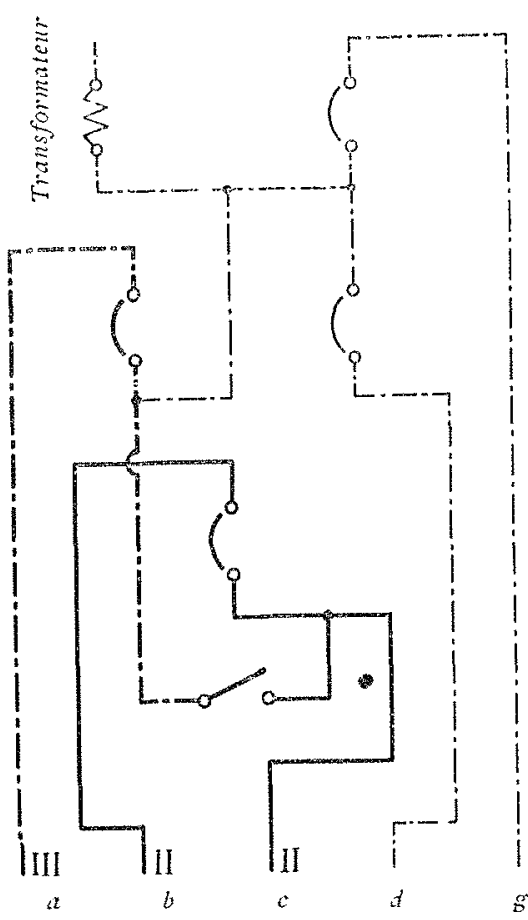

Fig. 8. - Schéma de montage du poste no 5 .

a. Feeder III venant de Mallifaud. $b$. Feeder II venani de Mallifaud.

c. Feeder II allant au poste 10.

d. Câble allant au poste 6.

g. Câble allant au poste 1 .
Le quatrième feeder est destiné plus spécialement à l'alimentation d'un quartier industriel, le cours Berriat ; c'est le plus étendu de tous; il est complètement séparé des 3 premiers. Ces 4 directions constituent le réscau urbain.

Les cinquième et sixième feeders sont aussi séparés des autres ct ne peuvent être reliés ensemble; ils distribuent l'énergie dans la banlieue de Grenoble, et sont, par conséquent, de moins grande importance que les 4 premiers; ils constituent le réseau suburbain. Le septieme panneau de feeder est réservé actuellement à l'éclairage de la sous-station.

Tous ces feeders sont établis en câbles armés souterrains système Berthoud-Borel. Leur section est de $3 \times 60 \mathrm{~m} / \mathrm{m}^{2}$ pour les 4 premiers, et de $3 \times 20^{\mathrm{m} / \mathrm{m}^{2}}$ pour les 2 autres. Ces câbles sont revêtus de deux gaînes de plomb et de deux rubans d'acier de I $\mathrm{m} / \mathrm{m}$ d'épaisseur, enroulés dans le mème sens et à joints recouverts. Une couche de jute goudronné est interposé entre le plomb et le fcuillard. Ils sont recouverts extérieurement d'une enveloppe protectrice en toile grossière asphaltée. Les différents conducteurs d'un même câble peuvent être facilement distingués du fait qu'ils sont recouverts d'une enveloppe différente (toile fine, toile grossière, papier). Les câbles sont isolés au papier imprégné ; l'épaisseur de la couche isolante entre les conducteurs est environ de $4,5 \mathrm{~m} / \mathrm{m}$, et entre les conducteurs et le plomb de 5,5 milimetres.

Les câbles ont été placés dans des tranchées, à 1 mètre de profondeur environ sous-chaussée, et à o mètres 60 environ sous trottoirs; ils reposent sur un lit de sable de $\mathrm{O}^{\mathrm{HI} I \mathrm{I}}$ et sont recouverts d'un deuxième lit de sable de $\mathrm{O}^{\mathrm{m}} \mathrm{I} 5$, sur ce lit est placé une rangće de briques jointives, sur ces briques om2o de terre criblće, puis le remblai, et enfin le pavage. Dans les traversées d'égouts ou de rues, où il n'a pas été possible de les mettre à la profondeur réglementaire, on les a placés dans des tuyaux de fonte de diamètre suffisant pour qu'ils puissent y passer librement.

Les boîtes de jonction, d'obturation et de dérivation sont en fonte, et peuvent recevoir une certaine quantité de matière isolante destinée à séparer complètement la partie intérieure du câble de l'air extérieur (cette matière isolante est un mélange de résine, de paraffine et d'huile).

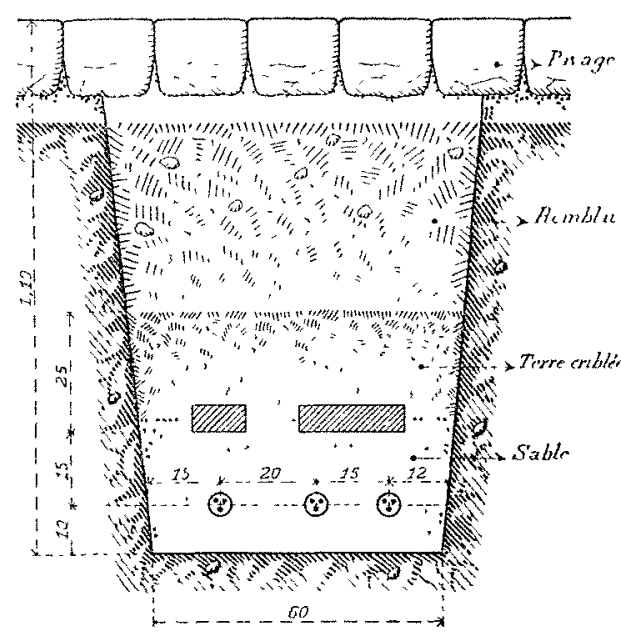

FIG. 9. - Coupe d'une tranchée.
Tous ces câbles ont été essayés en usine et soumis à une tension de $\mathrm{I} 5000$ volts, pendant 30 secondes, entre cordes et entre cordes et la terre; les essais d'isolement au galvanomètre ont aussi été effectués en usine.Ils ont donné un isolement kilométrique moyen de 5000 mégohms. Après la pose, tout le réseau a été soumis à une tension de 7500 volts pendant quelques heures; de nouveaux essais d'isolement ont, de même, été faits sur place. L'isolement kilométrique minimum garanti après la pose est de 750 mégohms.

La chute de tension prévue dans les feeders est, en moyenne de 1,5 pour ıoo. Tous ces câbles sont garantis pendant 3 ans par le constructeur.

Le réseau primaire présente cette particularité que la plupart des postes sont embrochés les uns à la suite des autres, c'est-à-dire que chaque poste est commandé par le précédent, de sorte qu'en cas de court-circuit, ou d'avarie, sur un câble, ou dans un poste de tranformateurs, seule la partie du réseau située en aval de l'accident est privée de courant.

Cette disposition présente de même un grand avantage lorsque l'on a à effectuer des branchements sur un câble primaire ; de cette façon, on peut, en effet, sectionner la partie intéressée sans interrompre tout un feeder, et sans être obligé de faire exécuter la manœuvre depuis la sousstation.

Lorsqu'une dérivation importante se trouve située à proximité de la sous-station et loin d'un poste de transformateurs, on place alors des boîtes de coupures établies pour la tension d'exploitation de 5000 volts, et renfermant des broches à coupe-circuits, amovibles et immergées dans l'huile. Cette disposition remplace les coupe-circuits des postes transformateurs; tout en étant moins pratique elle donne des résultats assez satisfaisants; elle présente surtout l'avantage d'être économique.

Postes de transformateurs. - Le modèle type d'un poste de transformateur renferme du côté haute tension:

2 boîtes d'extrémité de câbles haute tension $B_{1 \mathrm{l}}$.

3 coupe-circuits tripolaires haute tension, à huile $C_{h}$.

I poste-tripolaire de limiteurs de tension $L_{l}$ du côté basse tension.

I boîte d'extrémité de câble basse tension pour l'éclairage particulier $B_{b}$.

Un certain nombre de boîtes d'extrémité basse tension pour l'éclairage public $B_{p}$.

I interrupteur tripolaire basse tension éclairage particulier $I_{p}$.

I interrupteur tripolaire basse tension éclairage public $I_{r}$. 
I tableau de coupe-circuits des câbles de l'éclairage public $\mathrm{C}_{\mathrm{p}}$.

I appareil de mise à la terre $\mathrm{C}$.

I commutateur de phases et de terre avec lampe témoin $I_{t}$

Et enfin 1 transformateur $T$.

Le câble haute tension " feeder 》 est relié directement à ces trois postes de coupe-circuits. L'un d'eux commande la direction du poste suivant, l'autre les limiteurs de tension, et enfin le troisième, le transformateur du poste, placé dans le haut de la tourelle pour le réseau urbain, et sur le seuil du poste pour les réseaux périphérique et suburbain. Ces coupe-circuits sont du modèle Sprechler et Schuler décrit précédemment, ainsi que les limiteurs du modèle Vedovelli.

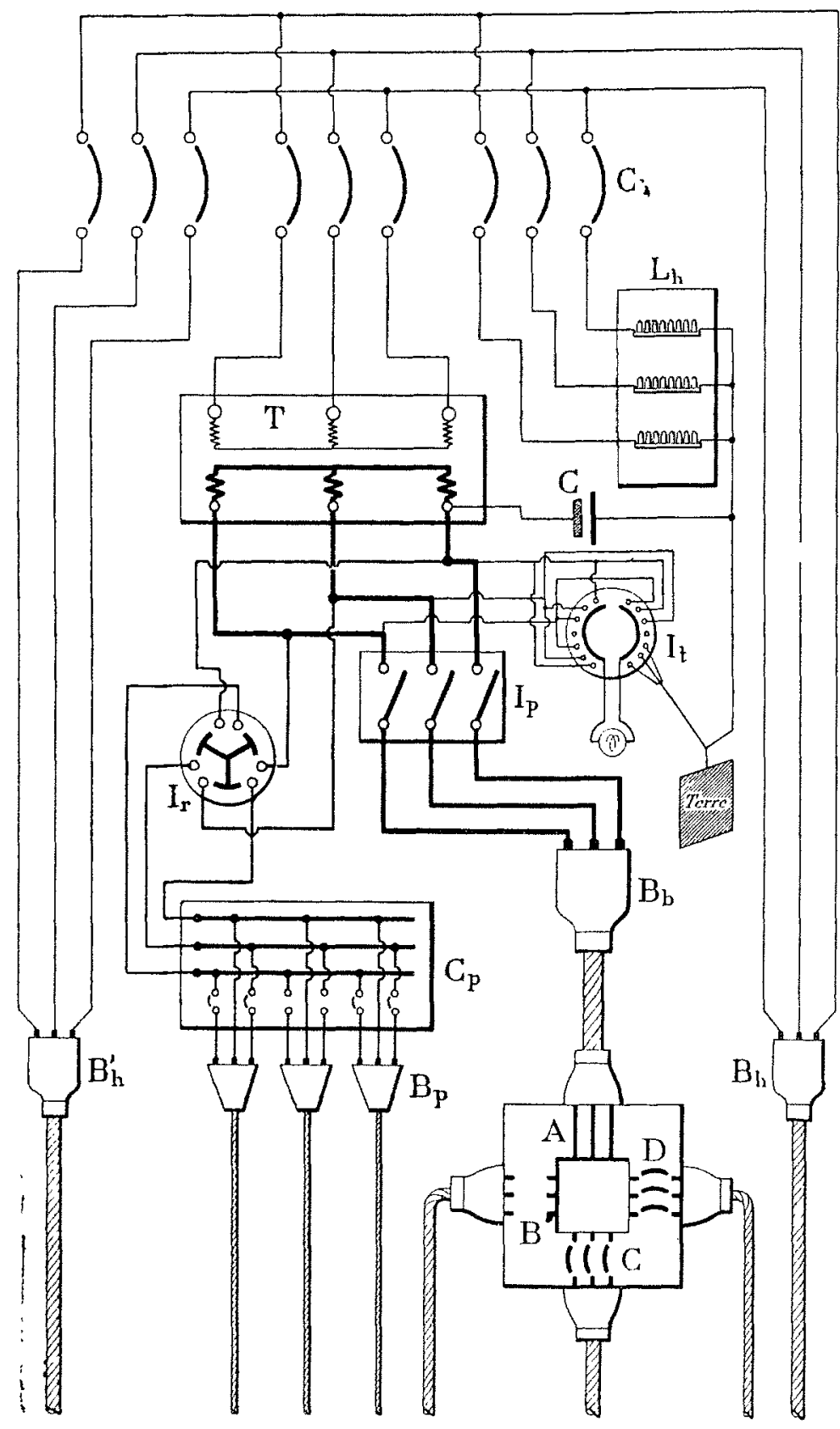

Frg. 10. - Schéma du montage type d'un poste de transformation.

Le courant basse tension venant du transformateur est réparti immédiatement dans deux directions: l'éclairage particulier et l'éclairage public.

L'interrupteur tripolaire basse tension de l'éclairage particulier permet de fermer le courant directement sur la boîte d'extrémité basse tension du réseau particulier, qui, elle, conduit le courant à une boîte de répartition placée dans la chaussée à quelques mètres du poste.
L'interrupteur tripolaire basse tension rotatif de l'éclairage public est mancuvrable depuis l'extérieur du kiosque; il permet de fermer le courant sur le tableau de coupe-circuits de l'éclairage public qui, lui-même, est relié aux différentes boîtes d'extrémité du réseau public.

L'appareil de mise à la terre est pris en dérivation sur l'une des bornes secondaires du transformateur; il est constitué par deux plaques de laiton isolées, l'une de l'autre

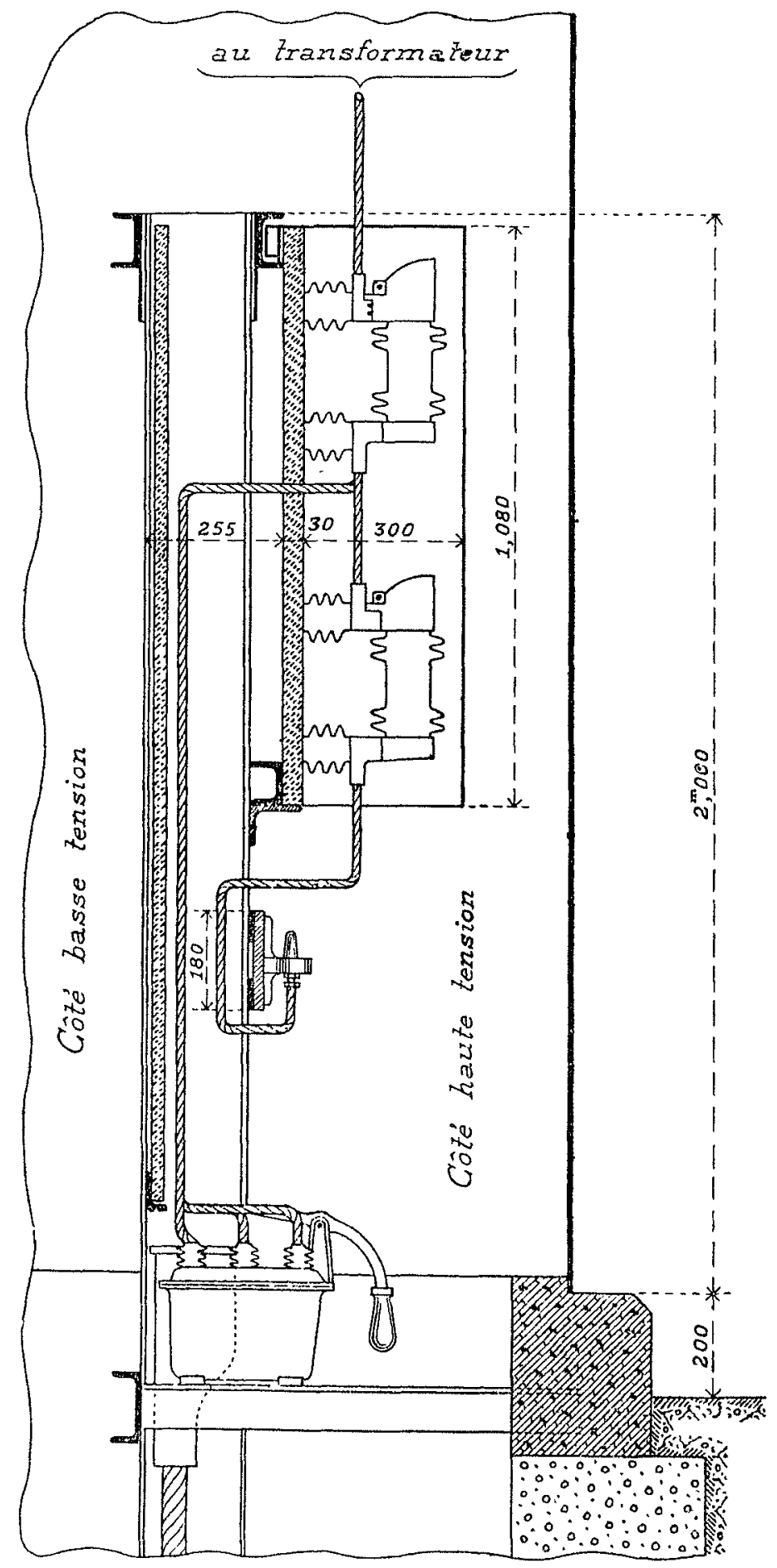

FIG. Ir. - Détail d'un transformateur fusible, coupe-circuits et appareil de mise à la terre.

par une feuille de papier paraffiné; l'une d'elles est reliée au réseau et l'autre à la terre. L'appareil est réglé pour fonctionner à un survoltage de 100 pour 100 , soit à 250 volts.

Le commutateur de phases permet de mesurer approximativement le voltage au moyen d'une lampe sur chaque pont, et entre chaque phase et la terre $[(\mathrm{I}-2),(2-3),(\mathrm{I}-3)$, $(I-t),(2-t),(3-t)]$. Il sert surtout comme indicateur de terre. 
L'appareillage basse tension a été construit par la maison Hartmann et Braun de Frankfort.

Ainsi que nous le disions plus haut, le transformateur est placé dans la partie supérieure du poste où il repose sur la charpente métallique du tableau.

Les kiosques urbains affectent la forme d'une tourelle de section circulaire de $\mathrm{I}^{\mathrm{m}} 5 \mathrm{o}$ de diametre et de $4^{\mathrm{m}} 5 \mathrm{o}$ de hauteur. Ils reposent sur un socle de béton dans lequel on a ménagé des caniveaux pour le passage des câbles haute et basse tension. Ces kiosques sont fixes; on accède aux tableaux des appareils haute et basse tension par l'intermédiaire de deux portes placées vis-à-vis de ceux-ci. Du côté de la basse tension la porte emprunte toute la hauteur de l'édifice, ce qui permet, par conséquent, l'enlèvement et la mise en place des transformateurs.

Ces appareils ont été construits par la Société " L'Eclairage Electrique $\gg$ de Paris; ils sont du type trıphasé à trois colonnes conjuguées à l'air libre; le rapport de transformation à vide est de 5 ooo à 125 volts (tension composée); leur puissance cst exprimće en $\mathrm{KVA}$ pour $\cos \varphi=0,7$, et ils doivent pouvoir supporter une surcharge permanente de I $5 \%$ sans avarie.

Des essais de rupture des isolants ont été faits en usine à une tension 10000 volts entre le primaire et la masse, et entre le primaire et le secondaire, et à une tension de I 500 volts entre le secondaire et la masse.

Les rendements en pleine charge, les consommations à vide, et les chutes de tensions pour $\cos 0=\mathrm{I}$, et pour $\cos p=0,7$, sont indiqués sur les courbes de la figure $\mathrm{r} 3$, pour les différentes puissances, qui varient de 3,7 à 150 kilovoltampères.

L'élévation de la température au-dessus de l'ambiante, après I $2_{2}$ heures de fonctionnement à pleine charge, ne dépasse pas $4^{\circ}$ pour le cuivre et $55^{\circ}$ pour le fer.

Réseau secondaire urbain souterrain. - Les postes de transformateurs dont nous venons de parler (tourelles métalliques) alimentent exclusivement des cables souterrains. Ceux-ci sont de deux sortes: ceux de l'éclairage public et ceux de l'éclairage particulier.

Eclairage mublic. - Les câbles d'éclairage public partent séparément du poste et rayonnent tout autour de celuici; la plupart sont réunis directement à d'autres postes du même feeder, de sorte qu'ils peuvent ètre alimentés indifféremment par l'un des deux transformateurs, auxquels ils aboutissent, ou par les deux simultanément, au cas où l'on voudrait boucler le réseau; d'autres constituent des dérivations.

Les grandes artères de la ville possèdent une double rangée de câbles, de façon à éviter autant que possible les traversées des rues. Lorsqu'il en est ainsi, on alimente généralement le côté pair avcc un transformateur, et le côté impair avec un autre, de façon à ce que les rues jouissent au moins de la moitié de l'éclairage ordinaire au cas où un accident se produirait sur l'un des transformateurs. Ces câbles, dont la section varie de $3 \times 20 \mathrm{~m} / \mathrm{m}^{2}$ à $3 \times 7,5 \mathrm{~m} / \mathrm{m}^{2}$, alimentent exclusivement les lampes à arc et à incandescence de l'éclairage public et les éclairages d'escaliers. Nous avons déjà signalé que les interrupteurs qui les commandent sont manœuvrables depuis l'extérieur du poste au moyen d'une clé à douille spéciale que possèdent seuls les allumeurs.

Ce sont des câbles armés du même système que ceux à 5000 volts (Berthoud-Borel), mais etablis pour un voltage inférieur; ils sont essayés à I 500 volts en usine. Leur revêtement extérieur est constitué par une toile fine asphaltée. Les boìtes de dérivation et de jonction sont du même modèle que celles des câbles à haute tension, mais de plus faibles dimensions.

Eclairage particulier. - Les câbles d'éclairage particulier sortant des postes de transformateurs vont aboutir, ainsi qu'il est indiqué plus haut, à des boîtes de répartition placées dans le sol; ces boîtes, de forme rectangulaire, dont l'étanchéitć est assurée par une double fermeture et un joint en caoutchouc sur un des quatre côtés, renferment

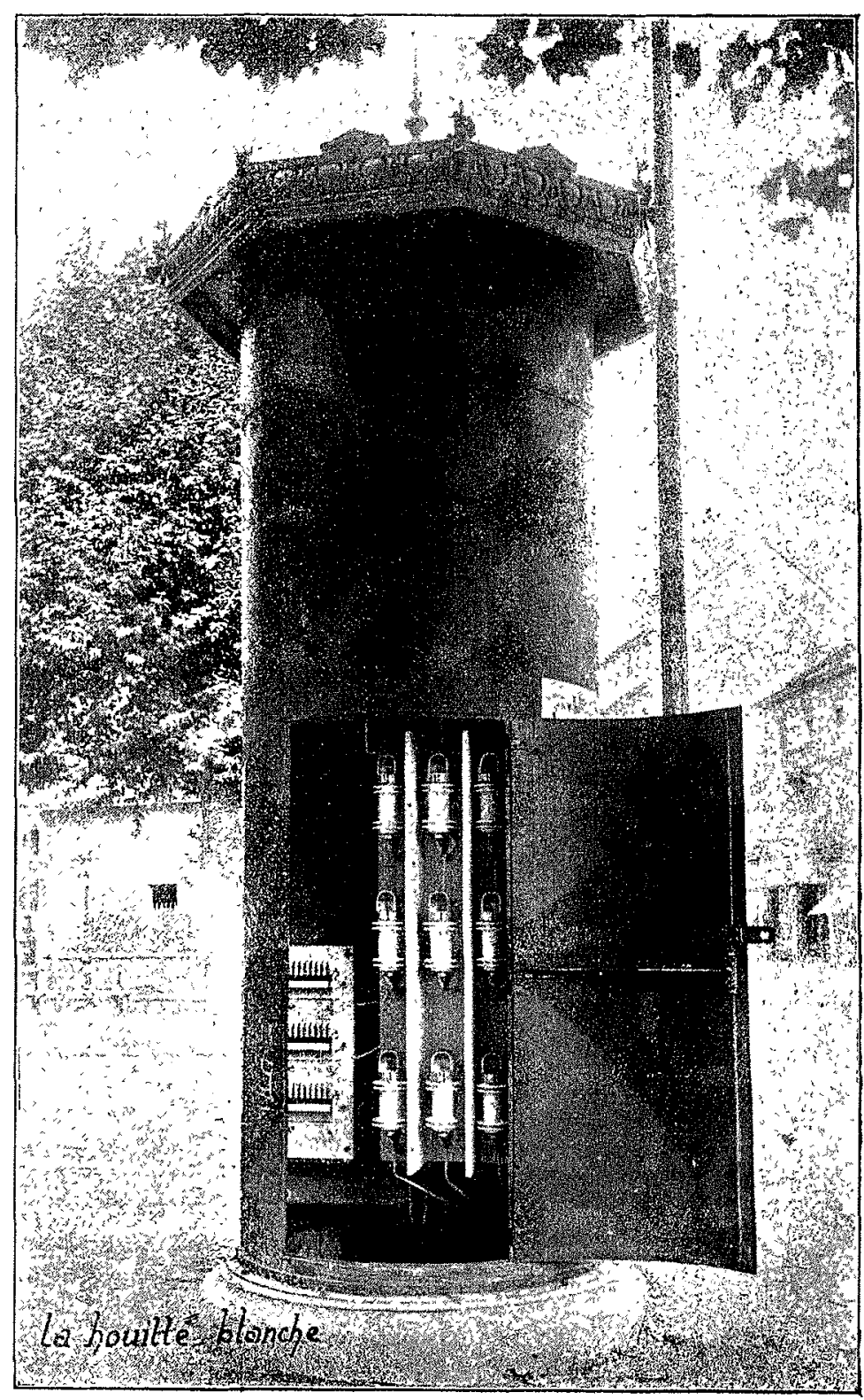

FIG: 12. - Vue d'un transformateur. Côté haute tension.

trois barres ou viennent aboutir le câble du poste. Les trois autres directions restant libres communiquent avec ces barres par des coupe-circuits amovibles à manettes porcelaine; à ces trois directions sont réunis des câbles d'éclairage particulier qui rayonnent autour du poste. Suivant l'importance du quartier où se trouve monté le transformateur, il y a $\mathrm{I}, 2$ ou 3 boîtes de répartition reliées à des boittes d'obturation dans le poste.

De même que cela a lieu pour l'éclairage public, les câbles partant des boîtes vont, pour la plupart, rejoindre d'autres boîtes reliées à d'autres transformateurs de même feeder; d'autres constituent de simples dérivations. 
Les mêmes manœuvres de répartition et de couplage que nous avons indiquées pour l'éclairage public peuvent done être effectuées pour l'éclairage particulier.

A signaler que, dans les grandes artères comprenant un double réseau, le côté pair est alimenté par un transformateur et le rôté impair par un autre, pour les raisons données ci-devant:

Ces câbles, dont la section varie de $3 \times 150 \mathrm{~m} / \mathrm{m} 2$ à $3 \times 25 \mathrm{~m} / \mathrm{m}^{2}$, ont été construits par la maison Grammont; ils sont du type dit "armé », et ont été soumis en usine à une tension de I 500 volts. Leur isolement kilométrique a été mesuré de même que ceux de Berthoud-Borel, en usine et sur place. En résumé, leur structure diffère peu de celle des câbles de Berthoud-Borel. Ils sont revêtus cxtéricurement d'une couche de jute.

Sur ces câbles sont branchés les petits moteurs d'une puissance égale ou inférieure à $3 \mathrm{HP}$, et l'éclairage des particuliers.

Les boîtes de jonction et de dérivation sont en fonte et d'un modéle analogue à celui des autres constructeurs.

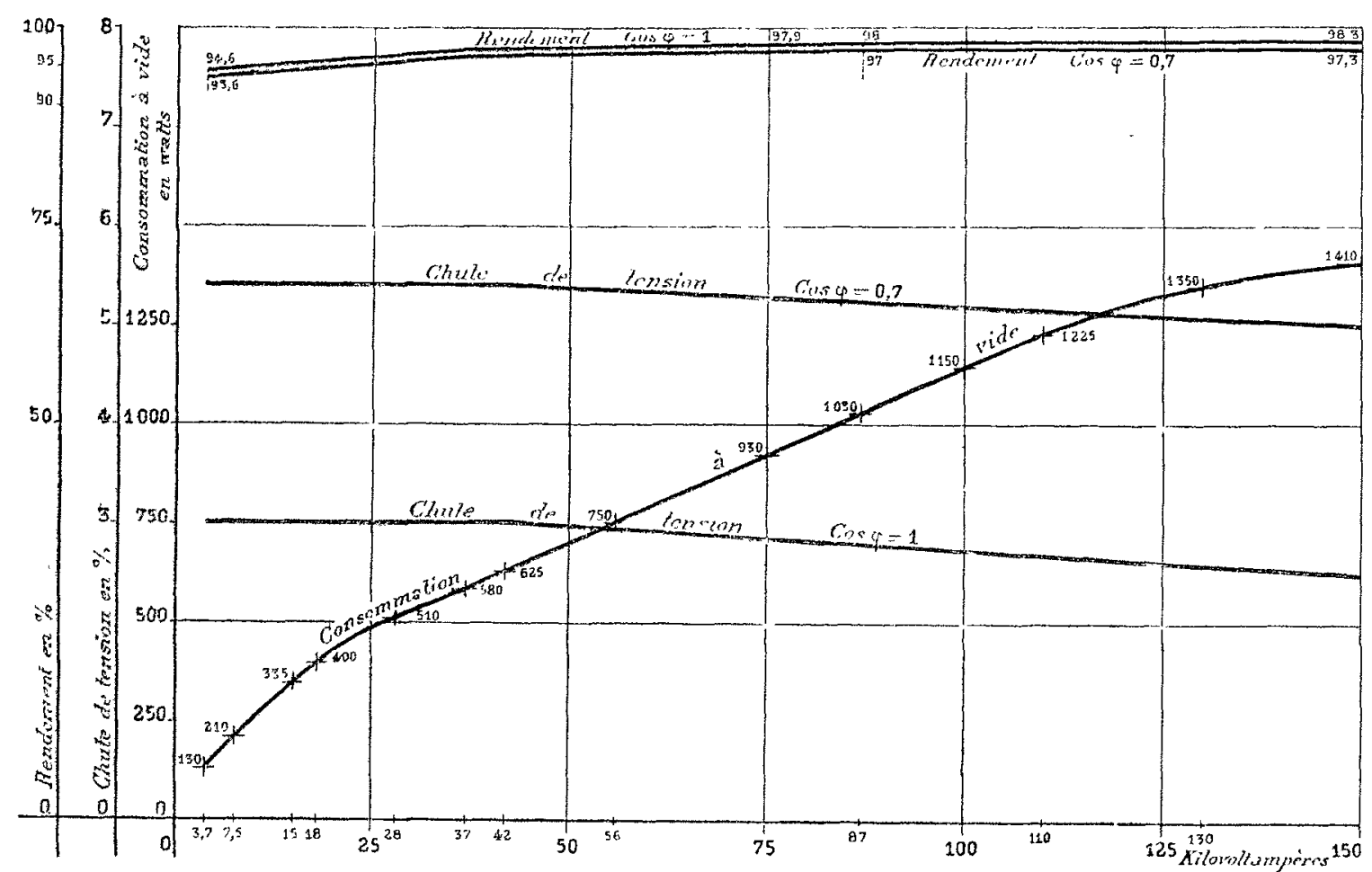

Fig. 13. - Courbes de consommation à vide, de chute de tension et de rendement.

Avant de lâcher les câbles, notons encore qu'ils ont été calculés pour une chute de tension de 3 pour too.

Branchements. - Les branchements des immeubles sont exécutés avec du câble dont la section varie de $3 \times 35 \mathrm{~m} / \mathrm{m}^{2}$ à $3 \times 10 \mathrm{~m} / \mathrm{m}^{2}$ pour l'éclairage particulier, et en $2 \times 5 \mathrm{~m} / \mathrm{m} 2$ pour l'éclairage des escaliers. Ces câbles aboutissent à des coffrets en fonte, formant bâti d'obturation, et scellés derrière les portes d'allées dans les immeubles; ils renferment des barettes de coupe-circuits amovibles. Les colonnes montantes viennent se raccorder à la partie supéricure du coffret.

Les branchements des lampes à arc sont en $2 \times 7,5 \mathrm{~m} / \mathrm{m}^{2}$; ils aboutissent dans le socle des candélabres, ou dans des coffrets en fer spéciaux, où la boîte d'obturation est remplacée par un tube en porcelaine dans lequel l'extrémité du câble est noyée dans de la matière isolante.

Les branchements des lampes àincandescence se font dans les mêmes conditions, avec cette différence que les câbles ont une section de $2 \times 2 \mathrm{~m} / \mathrm{m}^{2}$ et que les tubes porcelaine sont de plus faibles dimensions.
Quant aux transformateurs des abonnés de force motrice d'une puissance supérieure à $3 \mathrm{HP}$, ils sont réunis aux feeders par l'intermédiaire de boites d'obturation haute tension dont il a été question pour les postes urbainsmunicjpaux. Les tranchées renferment donc généralement I, 2 ou 3 câbles dont 1 haute tension et 2 basse tension.

Réseau périphérique. - Dans ce réseau, lcs kiosques de transformateurs, au nombre de 18 , affectent une forme rectangulaire de im.5o sur $\mathrm{I}$ m., et sont établis en ciment armé; ils sont munis de trois ouvertures, dont une de chaque côté du poste et sur la plus petite face, donnant accès aux tableaux haute et basse tension, et une autre sur le devant, permettant les mancuvres du transformateur placé au niveau du sol; c'est de même par cette ouverture que l'on accède le plus commodément aux différentes boîtes d'obturation.

Certains postes de transformateurs alimentent uniquement des lignes aériennes, d'autres alimentent des lignes souterraines et des lignes aériennes.

L'appareillage de ces pos. tes est le même que celui utilisé dans les tourelles métalliques; il est réparti un peu différemment, mais le principe reste le même. Le montage de ces postes, ainsi que celui des postes du réseau urbain, a été exécuté par la Société d'Applications Industrielles.

Les lignes souterraines alimentées par ces kiosques sont identiques à celles du réseau urbain, ainsi que tous leurs accessoires.

Les fils aériens sortent par la petite toiture supérieure du kiosque et sur les côtés de celle-ci; ils reposent sur des isolateurs à double cloche, de part et d'autre de la paroi, qu'ils traversent à travers des planchettes de chêne dontils sont isolés par des manchons en porcelaine; de là ils vont rejoindre un pylône en treillis métallique placé à quelques mètres du poste, d'où ils rayon. nent dans les différentes directions à desservir. De même que pour le réseau souterrain, il y a 6 fils, 3 pour l'éclairage public et 3 pour l'éclairage particulier. La section de ces fils varie de $44 \mathrm{~m} / \mathrm{m}^{2}$ à $5 \mathrm{~m} / \mathrm{m}^{2}$ pour l'éclairage particulier et de $15 \mathrm{~m} / \mathrm{m}^{2}$ à $7 \mathrm{~m} / \mathrm{m}^{2}$ pour l'éclairage public.

I a plupart du temps, les secondaires de plusieurs postes de transformateurs d'un même feeder peuvent être couplés ensemble suivant le principe du réseau souterrain. Dans ce cas, les boîtes visitables sont remplacées par des coupecircuits aériens, à broches amovibles, montés sur isolateurs et placés sur les consoles ou pylônes. Ces appareils sont construits de telle sorte que leur mancuvre est rendue possible, même en charge.

Généralement, ces coupe-circuits sont placés au départ des postes sur chaque direction, et au milieu des lignes, car celles-ci sont souvent très longues; de cette façon, la moitié de chaque direction est alimentée par un poste 
différent en service normal, et, en cas d'accident à un transformateur, la direction entière et même plusieurs peuvent être alimentées par un seul poste.

Les fils constituant ces lignes sont en bronze siliceux mi-dur dont le coefficient de rupture est de $45 \mathrm{kgs}$ par m $\mathrm{m} 2$, leur conductibilité est de 98 pour roo.

Les lignes sont établies sur des supports en fer, calculés chacun pour l'effort auquel ils doivent résister. Pour ceci, on a admis un effort maximum du vent de $150 \mathrm{kgs}$ par $\mathrm{m}^{2} \mathrm{de}$

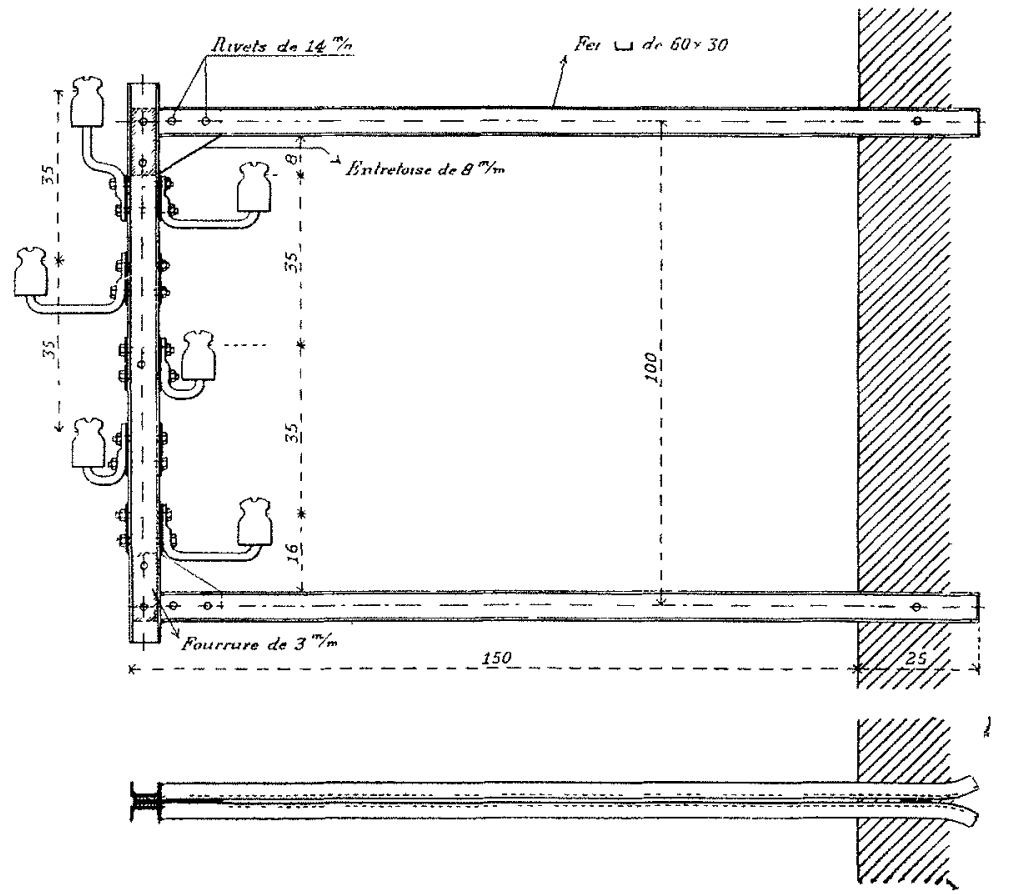

FIG. I4. - Elévation et plan d'une console d'alignement.

de surface offerte au vent, tant pour les supports que pour les fils; on a admis, en outre, que la tension maxima des fils ne dépasserait pas $8 \mathrm{kgs}$ par $\mathrm{m} / \mathrm{m}^{2}$ et on a fait travailler le fer à 7 kgs au maximum.

Les lignes reposent sur deux sortes de supports; dans les rues où les maisons riveraines sont suffisamment élevées,
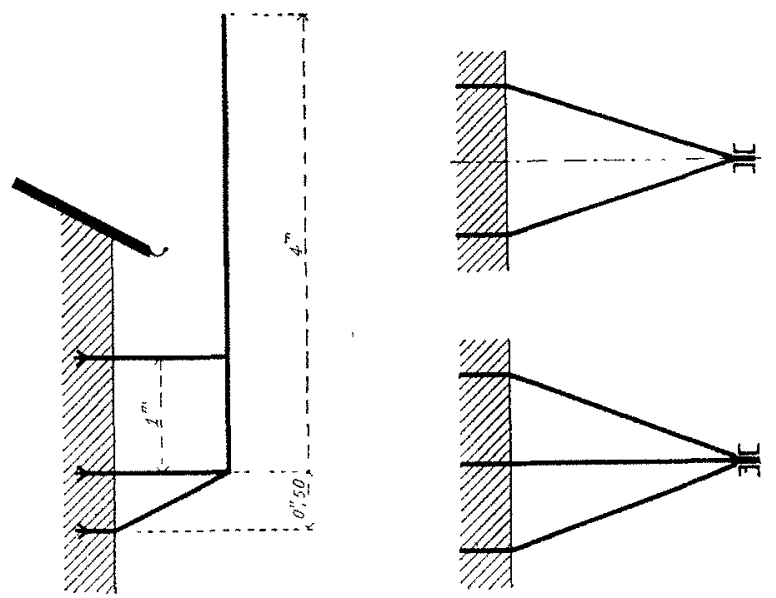

FIG. 15. - Types divers de consoles.

on a placé des consoles, et partout ailleurs des poteaux en treillis métallique.

Les consoles sont de 4 types différents: les consoles d'alignement; les consoles d'arrêt; les consoles à potelets d'alignement; les consoles à potelets d'arrêt.

Les consoles d'alignement sont établies en fers $U$ de $60 / 30$ juxtaposés dos à dos; les scellements ont $\mathrm{o}_{2} 5$ de profondeur, et la saillie totale extérieure est de $\mathrm{I}_{2} 5$; leur hauteur est de I $\mathrm{m}$.
Les isolateurs sont placés en quinconce à une distance verticale de o m. 35, et munis de tiges de différentes longueurs, ce qui rend presque impossible tout contact accidentel et facilite beaucoup les dérivations en ligne.

Les isolateurs sont du modèle Parvillé, type 102, à double cloche; ils sont scellés au soufre sur des tiges en fer galvanisé. Ces tiges sont fixées aux consoles par des boulons en fer galvanisé logés entre les 2 fers à $U$.

Les isolateurs, ayant de grands efforts à vaincre, sont d'un modèle supérieur, ainsi que leurs tiges.

Les poteaux d'alignement, en treillis métallique, sont construits en fer-cornières de $45 \times 45 \times 5$ pour les montants,

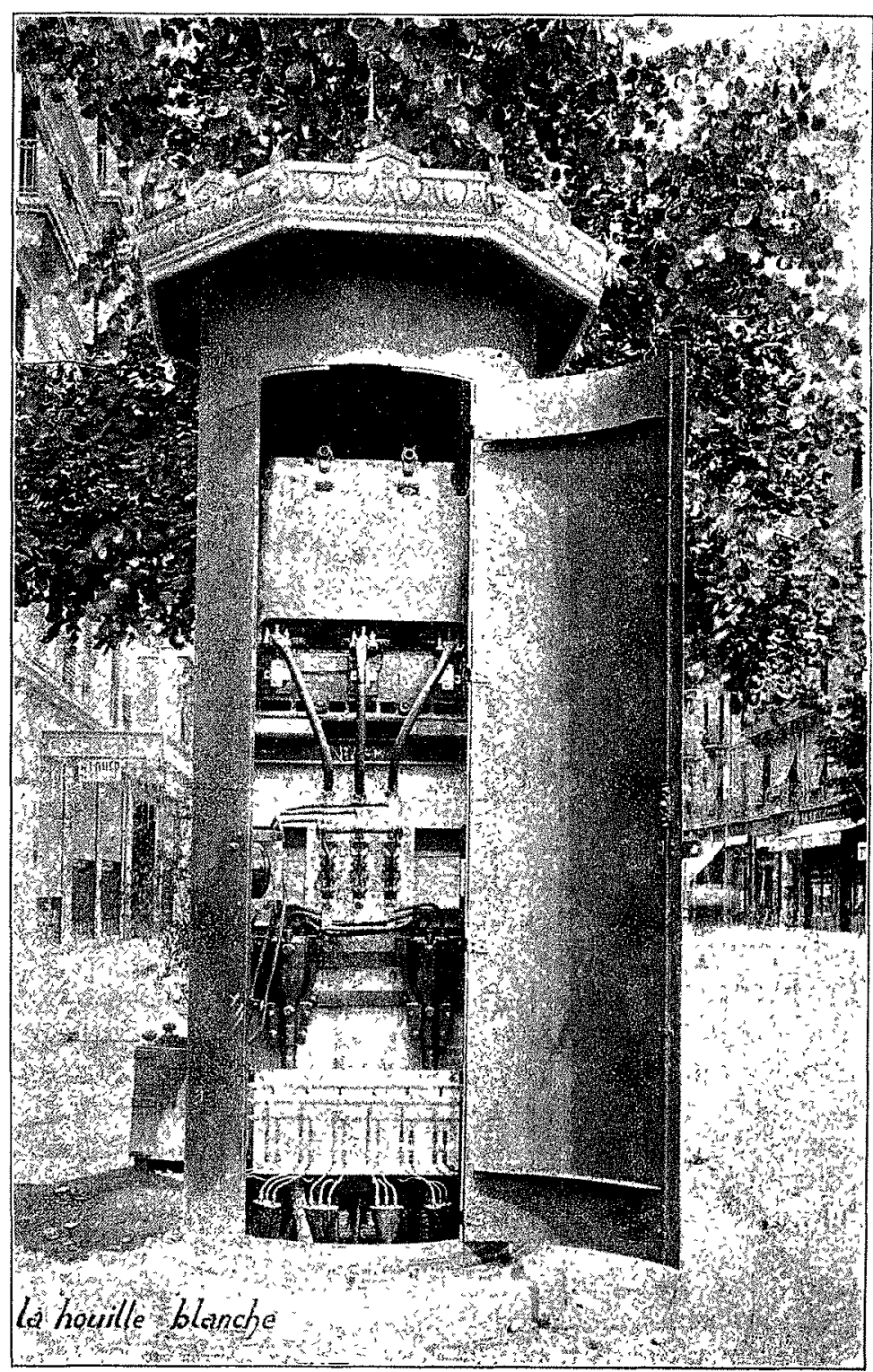

Fig. 16. - Vue d'un transformateur. Côté basse tension.

et de $30<<16 \times 3$ pour les croisillons. Leur poids est de I 60 kilogrammes. Ils sont encastrés dans un massif de béton de $\mathrm{I} \mathrm{m}^{3}$. environ; leur hauteur totale est de $9 \mathrm{~m}$. 5o; la profondeur d'encastrement étant de I m. 3o, il rest $8 \mathrm{~m} .20$ disponibles au-dessus du sol. Ils présentent une section quadrangulaire de o m. 3o à l'encastrement, et de o m. 15 au sommet. Les isolateurs sont fixés sur des plaques de tôle au moyen de boulons galvanisćs.

Les pylônes d'arrêt, ou d'angle, calculés suivant les données indiquées plus haut, ont une hauteur et des dimen. sions d'encombrement, à la base et au sommet, variant selon les besoins et les endroits oú ils sont placées. Certains 
d'entre eux ont une hauteur totale de $14 \mathrm{~m}$, une section rectangulaire à l'encastrement de $\mathrm{I}^{\mathrm{m}} \mathrm{I} O \times \mathrm{O}^{\mathrm{m}} 6 \mathrm{o}$, et pèsent environ $1200 \mathrm{~kg}$. Ils sont construits en cornières de $90 \times 90 \times 10$ et $70 \times 70 \times 8$ pour les montants, et $60 \times 60 \times 6$ pour les croisillons. Le cube de béton atteint alors 5 à $6 \mathrm{~m}^{3}$ suivant la nature du terrain, et la hauteur d'encastrement est de $200 \mathrm{~m}$. De tels supports ont à vaincre l'effort de 3 fils de $75 /$ ro et de 3 fils de $44 / 1$ o sous un angle de $90^{\circ}$ à $12 \mathrm{~m}$. de hauteur.

Les portées entre 2 supports consécutifs sont en moyenne de $35 \mathrm{~m}$.; elles varient entre 25 et $45 \mathrm{~m}$. suivant la nature et l'aspect du terrain. Les flèches ont été calculées de façon à ce que, par les plus grands froids, la tension des fils ne dépasse jamais $8 \mathrm{~kg}$. par millimètre carré.

Branchements. - Les branchements sur les lignes aériennes sont exécutés en fils nus sur isolateurs, jusqu'à hauteur et à proximité des portes des immeubles; à ces endroits, les fils sont arrêtés sur des isolateurs de forme spéciale qui permettent d'effectuer le raccordement des câbles sous-plomb des colonnes montantes à l'abri de l'humidité; de là, les câbles sous-plomb, logés dans des moulures de bois goudronnées, vont rejoindre des coffrets à coupecircunts amovibles d'un modèle analogue à ceux du réseau souterrain.

Ce genre de branchement étant trop coûtcux pour les installations de moyenne importance, on a adopté un autre système de coffrets étanches plus économiques, qui se placent à l'extérieur, près de la porte d'allée, et qui jouent le rôle des isolateurs spéciaux dits "Jacquet ", décrits ci-dessus et des coffrets. Ces coffrets sont surmontés d'une pipe double ou triple suivant le cas, et portent 2 ou 3 isolateurs à tenon, placés en-dessous de la pipe et sur lesquels viennent s'amorrer les fils. L'intérieur de ces coffrets renferme un coupecircuit, genre Edison, monté sur porcelaine. La partie supérieure de ce coupe-circuit est réunie aux isolateurs à tenon par du fil isolé à travers les pipes. La colonne montante se branche à la partie inférieure; elle peut donc ètre séparée du réseau en enlevant les bouchons des coupecircuits.

Les branchements de plus faible importance, tels que ceux de l'éclairage public et des petits abonnés, sont exécutés en fils nus de $16 / 10 \mathrm{~m} / \mathrm{m}$, placés sur des isolateurs à double cloche d'un modèle réduit; des ferrures spéciales à doubles tiges supportent les isolateurs. Le sous-plomb qui conduit le courant à la douille de la lampe est raccordé directement au fil nu.

Colonnes montantes et Installations intèrieures. Les colonnes montantes, destinées à l'alimentation des magasins et appartements des différents étages, sont établies dans les cages des escaliers, en fils ou câbles sous-plomb de 600 méghoms, logés dans des moulures; dans les traversées de paliers et murs, les moulures sont remplacées par du tube Bergmann armé. A chaque étage, la colonne vient aboutir à un coupe-circuit de branchement, genre Edison, sur lequel sont dérivés les abonnés. Afin d'éviter toute fraude, les fils des dérivations sont également renfermés dans une gaîne de plomb jusqu'au compteur.

Les compteurs sont placés sur des tableaux en bois dur, fixés sur des gros murs, dont ils sont espacés de 4 à $5 \mathrm{~cm}$; chaque tableau comporte un interrupteur omnipolaire à coupe-circuit. Les installations jusqu'à 20 lampes de 16 bougies, ou leur équivalent, sont branchées en monophasé; celles d'une importance plus grande sont alimentées en triphasé.
Réseau Suburbain. - Le réseau suburbain est alimenté par les feeders $V$ et VI dont nous avons dit un mot tout à l'heure; ils sont de moins grande importance que leurs frères urbains; l'un deux, toutefois, le VI, est très étendu, mais il ne distribue pas une quantité d'énergie bien considérable; leur section est de $3 \times 20 \mathrm{~m} / \mathrm{m}^{2}$. Ils ont été prévus assez largement, à cause de l'extension que prennent chaque jour les quartiers qu'ils traversent. Ils ont été placés comme au centre de la ville, et fournis par la même maison. Leur montage dans les postes de tranformation est de mêmeidentique à celui que nous avons décrit précédemment, c'est-à-dire que la plupart des postes sont embranchés les uns à la suite

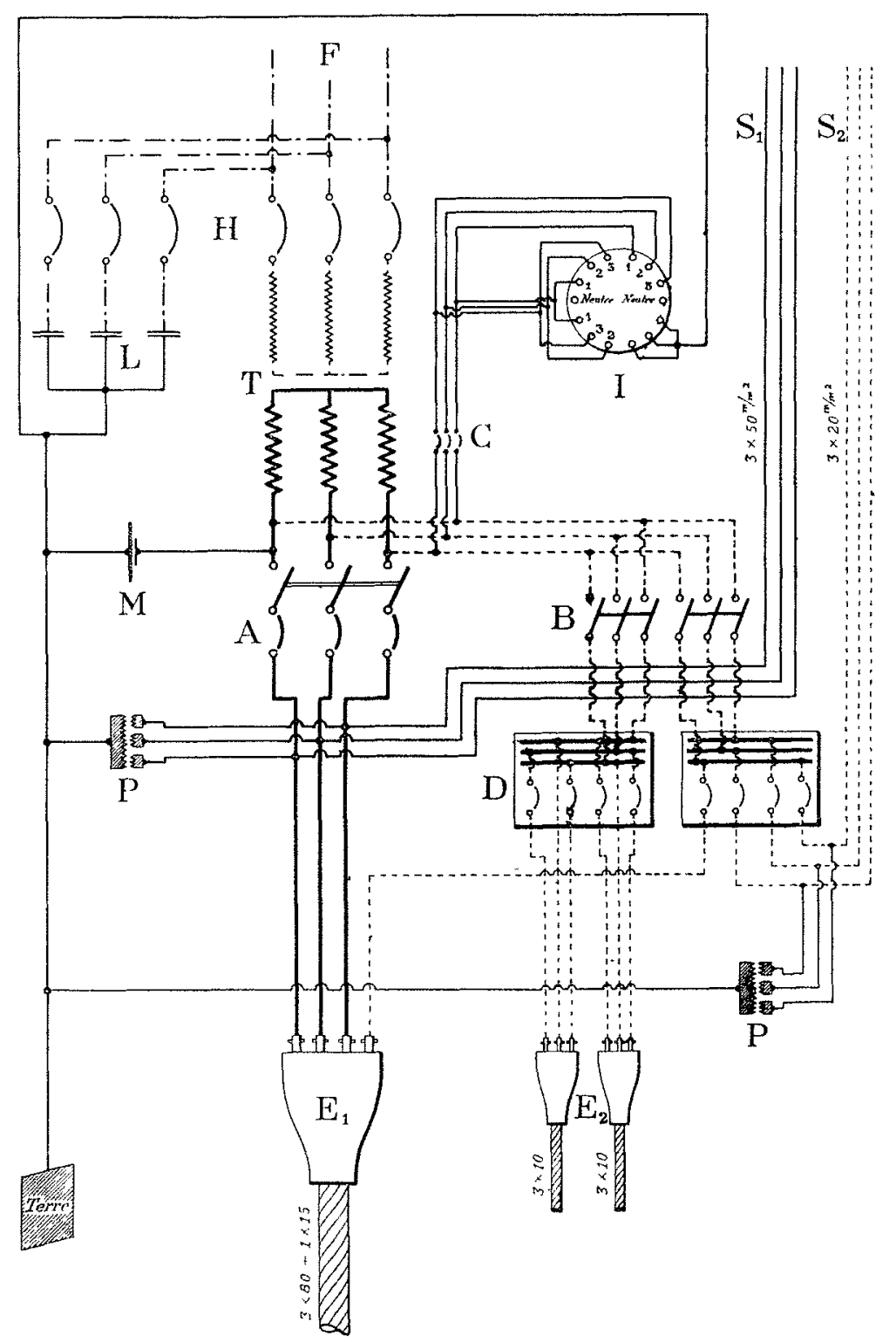

Fig. 17. - Schéma du montage d'un poste de transformateur du réseau suburbain.

des autres. Les kiosques de transformateurs sont de même modèle et ont les mêmes dimensions que ceux de la périphérie, mais, au lieu d'être en ciment armé, ils sont en tôle d'acier; de plus, ils présentent cette particularité que le poste lui-même sert de base au pylône de sortie et de répartition des lignes aériennes; cette disposition présente, outre l'avantage d'être plus économique, celui d'être beaucoup moins encombrante, car on évite de cette façon le pylône que l'on est obligé de placer à côté des postes en ciment armé.

Le montage intérieur des postes du réseau suburbain est sensiblement le même que celui des postes en ciment; il 
diffère surtout par la disposition des appareils, et par la présence de parafoudres à peignes basse tension $\mathrm{P}$, qui nous ont paru indispensables vu la grande longueur des lignes secondaires, dans des chemins souvent dépourvus de toute habitation, et qui, par conséquent, sont exposées à des décharges atmosphériques.

Certains postes ont des départs secondaires souterrains et aériens. Les câbles souterrains, de même structure que ceux du réseau urbain, et fournis par les maisons BerthoudBorel et Grammont, présentent cette particularité qu'ils renferment un quatrième conducteur de plus faible section que les trois autres et qui est utilisé comme fil de retour de l'éclairage public.

En effet, ce réseau n'est pas tout à fait établi sur le même principe que celui de la périphérie et du centre. Là, il n'y a pas 2 réseaux complètement séparés, pour le public et le particulier, mais seulement un réseau particulier renforcé d'un fil de retour d'éclairage public qui permet l'allumage et l'extinction depuis les postes de transformateur. Cette disposition est indiquée dans le schéma de la figure 18 .

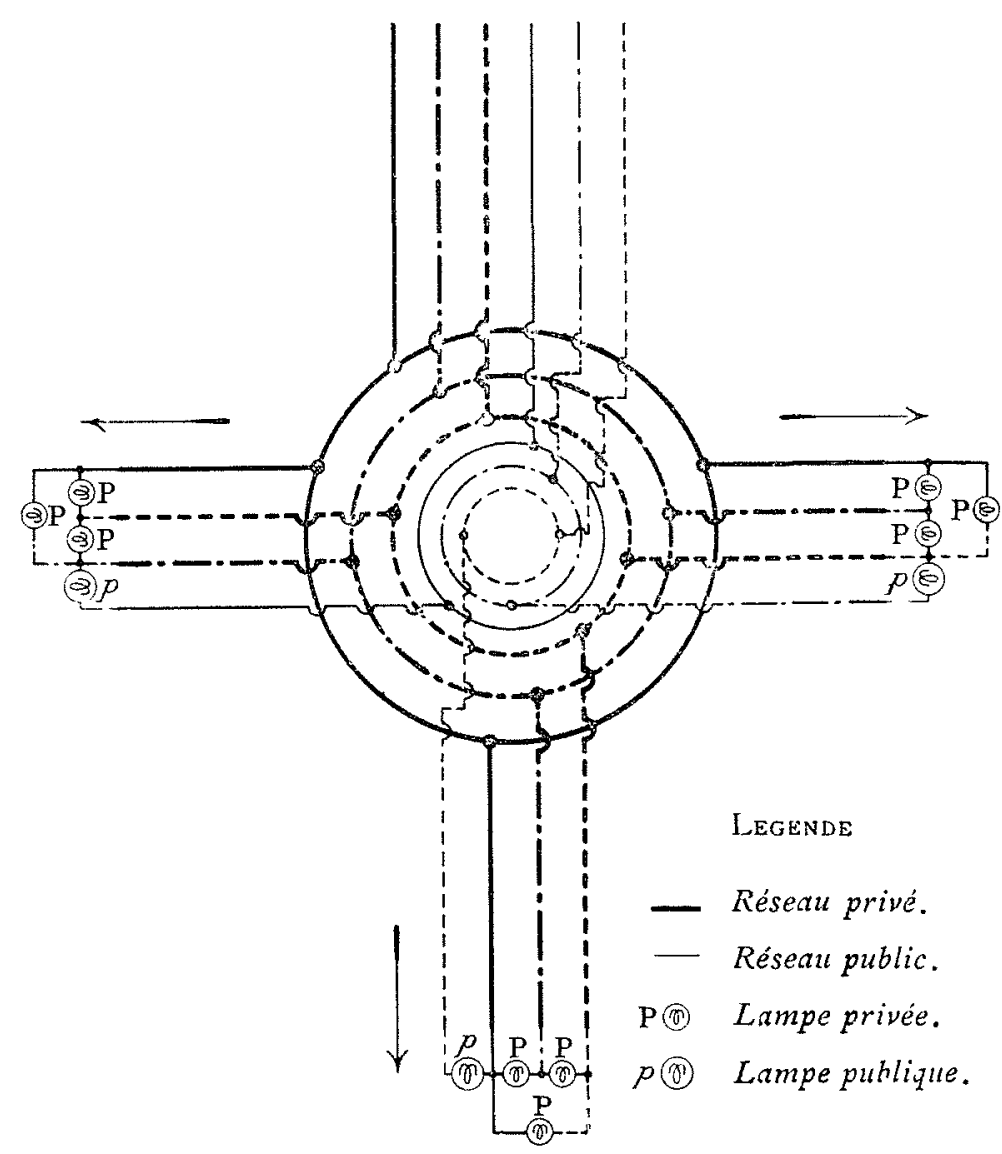

FIG, I8. - Schéma de la distribution du réseau suburbain.

Ce montage a été adopté par raison d'économie; il ne présente aucun inconvénient quant à la surcharge d'une des phases de l'éclairage particulier, parce que l'éclairage public est de peu d'importance dans la banlieue. Dans certains chemins, qui ne possèdent que fort peu de maisons, on a même établi la ligne en 3 fils ( $2 \mathrm{P}$ et $1 \mathrm{p}$ ); si plus tard la consommation du particulier vient à augmenter, on pourra, fort économiquement, remédier à la faiblesse de la ligne, en rajoutant simplement un quatrième fil. Dans d'autres chemins dépourvus de toute habitation on a placé seulement 2 fils ( $\mathrm{P}$ et I $\mathrm{p}$ ).

Les lignes sont construites en fils de bronze siliceux midur, comme en ville.

La section des lignes de l'éclairage particulier varie entre
$50 \mathrm{~m} / \mathrm{m}^{2}$ et $7 \mathrm{~m} / \mathrm{m}^{2}$, celle des fils de retour de l'éclairage public de 15 à $7 \mathrm{~m} / \mathrm{m}^{2}$. La chute de tension admise est en moyenne de $4 \%$ pour $\cos \varphi=0,8$. Ces lignes sont supportées par des poteaux de bois de sapin, injectés au sulfate de cuivre suivant le procédé de Bouchin. Toutefois, dans le angles et dans les points de répartition, on a placé des pylônes en treillis métalliques identiques à ceux du réseau périphérique.

Les poteaux de bois d'alignement ont une hauteur de $9 \mathrm{~m}$.; ils sont enfouis dans le sol de $\mathrm{I} \mathrm{m}$. 3o, et sont entourés à leur encastrement dans le sol d'une collerette en béton de ciment lent, de o m. 60 de hauteur et de o m: 25 d'épaisseur; la partie extérieure est terminée en forme de

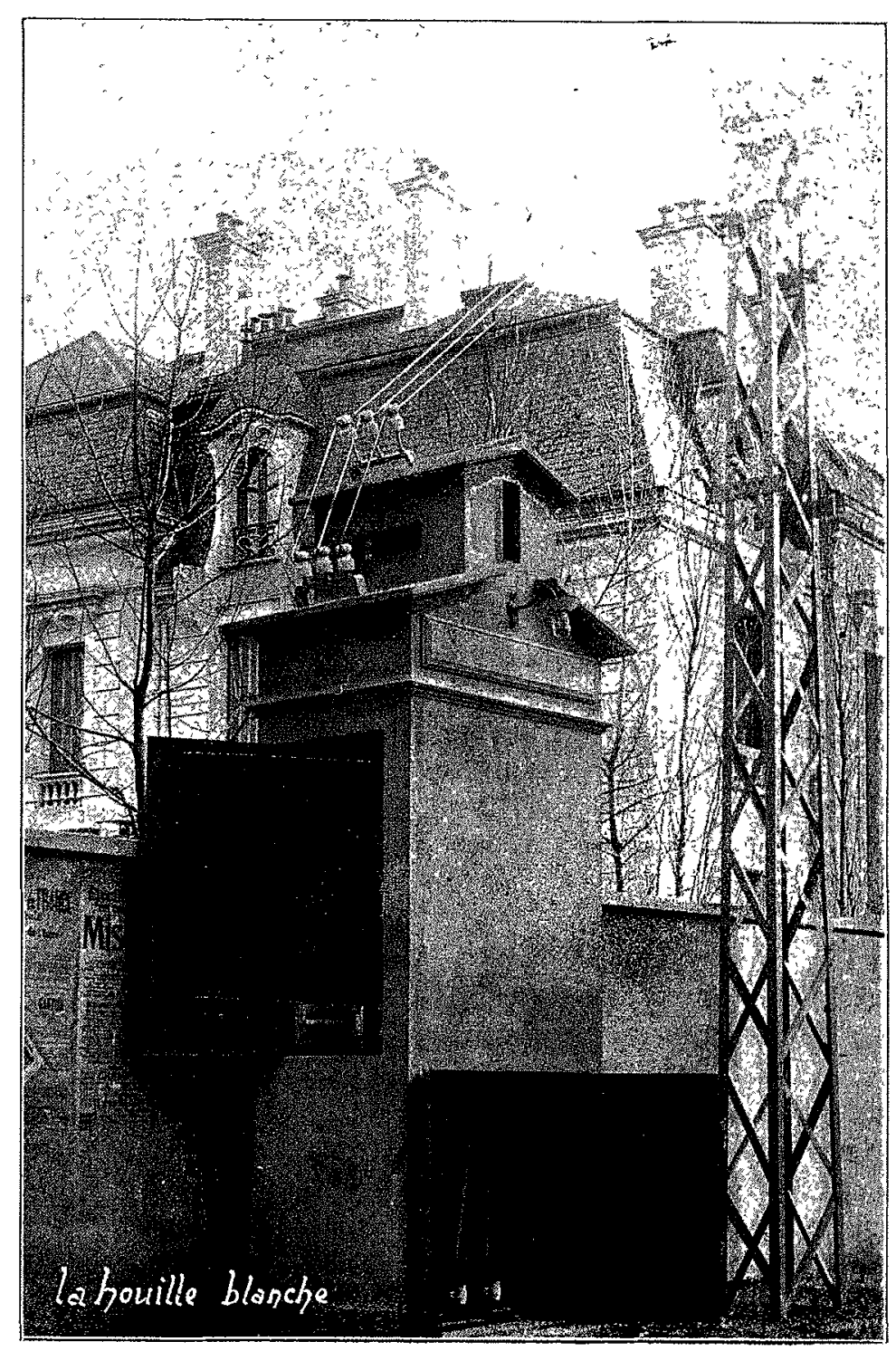

FIG. 19. - Vue d'un poste de transformateur du réseau périphérique.

glacis. En plus de ceci, la base est goudronnée sur une hauteur de I mètre. Cette double précaution nous a paru indispensable, car l'expérience a montré que la plupart des poteaux périssent à l'encastrement.

Les isolateurs sont les mêmes que pour les lignes de la périphérie; ils sont fixés aux poteaux par des boulons en fer galvanisés. Toutes les fois que la chose a été possible, on a prévu l'alimentation des lignes par 2 postes de tranformateurs; cette manœuvre est rendue possible par l'existence de coupe-circuits aériens amovibles de même modèle que ceux décrits plus haut. En temps ordinaire, les postes marchent séparément, mais ils pourraient fonc. 
tionner en parallèle si la section du cuivre devaient insuffisante ou si un transformateur venait à faire défaut.

Les branchements des abonnés et de l'éclairage public sont exécutés comme pour le réseau périphérique. Toutefois les lampes publiques fixées sur poteaux de bois, sont reliées à la ligne par du câble sous-plomb de 600 méghoms, logé dans une moulure de bois goudronné, vissée elle-même au poteau.

Le montage des postes de transformateurs du réseau suburbain, ainsi que les constructions des lignes aériennes du réseau périphérique et de la banlieue, ont été exécutés par la Société Grenobloise d'Electricité.

\section{RÉSUMÉ GÉNÉRAL}

Le secteur de Grenoble comprend donc 2 réseaux différents :

Le réseau urbain comprend le réseau du centre et celui de la périphérie. Celui du centre comprend 35 postes de transformateurs montés dans des tourelles métalliques, alimentant des lignes secondaires souterraines.

Le réseau périphérique comprend 18 postes montés dans des kiosques en ciment armé, alimentant des câbles secondaires souterrains, mais surtout des lignes aériennes.

Le réseau suburbain comprend i 2 postes montés dans des kiosques-pylônes en tôle d'acier, alimentant de même quelques lignes souterraines, mais principalement des lignes aériennes.

En debors de ceci, il existe encore 12 postes de transformateurs installés, soit dans des bâtiments communaux (Ecoles), soit chez de gros clients d'éclairage.

Le réseau municipal comporte donc actuellement 77 postes de transformateurs; d'après la description donnée précédemment, les allumeurs ont donc 65 rotatifs à manœuvrer, pour procéder à l'éclairage de tout le territoire de Grenoble.

Les abonnés d'une force motrice supérieure à $3 \mathrm{HP}$ ont chez eux des transformateurs qui sont leur propriété; ceux-ci sont actuellement au nombre de 35 .

La puissance totale des $77+35=112$ transformateurs reliés au secteur de Grenoble est environ de $5400 \mathrm{kw}$., représentant une consommation à vide de $70 \mathrm{kw}$. environ.

$\mathrm{Au} 30$ avril 1906, il y avait environ l'équivalent de 30000 lampes de to bougies installées chez les particuliers; à celles-ci, il convient d'ajouter environ 220 lampes à arc et I 600 lampes d'escaliers.

Les abonnés de force motrice, au nombre de 150 environ, absorbent une puissance d'environ i $200 \mathrm{HP}$ aux bornes des moteurs.

La puissance de ces moteurs varie à l'infini de I/ Io à ıoo HP. Sauf un seul, de $85 \mathrm{HP}$, qui est alimenté directement à $b$ ooo volts, ils fonctionnent tous à I 20 volts.

Les bâtiments communaux ont nécessité l'installation de 3500 lampes à incandescence et de 20 lampes à arc.

Le réseau d'éclairage public comporte environ r 500 lampes à incandescence de 16 bougies, et 225 lampes à arc. Ces dernières présentent un certain intérêt au point de vue des charbons qui sont minéralisés; ils sont dus à M. Blondel. La puissance lumineuse hémi-sphérique de ces lampes, dont la consommation moyenne est de 400 watts, y compris le rendement du transformateur, est de 2000 bougies environ. Elles fonctionnent à un ampèrage de 14 à 15 ampères, sous 29 à 30 volts; chaque lampe est branchée en dérivation sur le câble éclairage public par l'intermédiaire d'un petit transformatcur, dont le rapport de transformation est de $120 / 30$ volts environ en charge, et I $20 / 44$ volts à vide. Cette chute de tension, qui paraît énorme, est indispensable au bon fonctionnement de l'arc; on évite de cette façon des débits anormaux lorsque les charbons sont au calage.

Enfin, avant de vous rendre la liberté, je crois intéressant de vous indiquer encore les longueurs des différentes sortes de lignes constituant notre secteur municipal :

Câbles à 5 ooo volts.................. 3o kil. Câbles à 120 volts Eclairage particulier 3 fils..... 30 - - - public - 35 Lignes aériennes I 20 volts Eclairage particulier 3 fils. $20-$ Lignes aériennes - - - public - 24 Câbles à 120 volts banlieue, 2,3 et 4 fils....... 7 -

J'espère, Messieurs, que ces explications, qui ont peutêtre été un peu longues, vous auront donné une idée du Service Electrique de la Ville, que nous nous efforcerons sans cesse de perfectionner, car notre plus ardent désir est de faciliter la tâche de ces vaillants industriels qui font la réclame et la gloire de cette Ville de Grenoble, qui est à la tête des cités de lumière, marchant si courageusement au progrès, et concourant ainsi à la prospérité et à l'honneur de la nation française.

\section{ÉTUDE SUR LES}

\section{BARRAGES EN MAÇONNERIE \\ et Murs de Réservoirs \\ (suite)}

\section{Calcul des barrages a profil triangulaire}

Parmi tous les profils que l'on peut imaginer, c'est celui dont le parement amont est vertical qui, à volume égal, donne en charge les meilleures conditions de stabilité; ou, inversement, et à conditions égales de stabilité, c'est celui

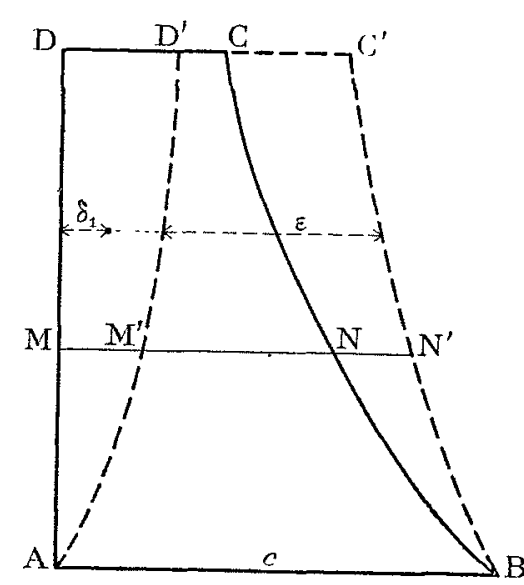

Fig. 69. qui est le plus économique. Ceci résulte immédiatement des considérations suivantes :

Soit $A B C D$ et $A B C$ 'D' deux profils ayant même hauteur, et, pour chaque section horizontale telle que $\mathrm{MN}$, même largeur, ce qui entraîne par suite même volume. Désignons par $N$ le poids des maçonneries de l'un et l'autre profil, par $\delta$ et $\delta$ ' les distances à la verticale AD des centres de gravité des deux profils, $\operatorname{par} p$ le poids de l'eau correspondant au triangle ADD' dont le centre de gravité est à une distance $\delta_{1}$ de la verticale $\mathrm{AD}$, et par $e$ la largeur de la base $\mathrm{AB}$. Les pressions $n_{v}$ pour le profil $\mathrm{ABCD}$, et $n_{\imath}$ pour le profil $\mathrm{ABC} I$ '), ont pour valeur:

$$
\begin{aligned}
& n_{v}^{\prime}=\frac{N}{e}+6 \frac{M_{2}}{e^{2}}-\frac{y^{3}}{e^{2}}=\frac{2 N\left(2 e-3 \delta^{\prime}\right)}{e^{2}}-\frac{y^{3}}{e^{2}} \\
& n_{i}^{\prime}:=2 \frac{N+p}{e^{2}}\left(2 e-3 \frac{N \delta^{\prime}+p \delta_{1}}{N+p}\right)-\frac{y^{3}}{e^{2}}
\end{aligned}
$$

